\title{
Frontline experiences and perceptions of Urgent Dental Care centre staff in England during the COVID-19 pandemic: a qualitative study
}

\author{
Anastasios Plessas, ${ }^{1}$ Martha Paisi, ${ }^{1}$ Rebecca Baines, ${ }^{2}$ Hannah Wheat, ${ }^{3}$ Maria Bernardes Delgado, ${ }^{1}$ Ian Mills ${ }^{1}$ and \\ Robert Witton*1
}

\section{Key points}

Participants reported that contributing meaningfully during COVID-19, by applying their dentistry skills to help those at need, was a valued and beneficial experience.

Reported negative experiences from
working during COVID-19 were
multifactorial stress and anxiety;
these emotions were predominantly
caused by concerns over safety and
operational UDC challenges, which
included fragmented communication
and guidance, poor PPE availability and
overwhelmingly high workload.

Team-developed and implemented strategies to enhance team unity during COVID-19 were viewed as key to maintaining UDC staff resilience.
Sustained efforts to support and improve dental teams' mental health and wellbeing are needed, along with more effective integration of dentistry into wider healthcare infrastructures to improve communication and patient care.

\begin{abstract}
Introduction Following the World Health Organisation declaration of COVID-19 as a global pandemic, routine dental care in the UK ceased, and Urgent Dental Care centres (UDCs) were established to offer remote and face-to-face urgent dental treatment for those in need.
\end{abstract}

Aim To explore perceptions and psychosocial experiences of frontline staff providing care at UDCs in England during COVID-19.

Method A qualitative research study using a phenomenological approach. Semi-structured interviews were conducted remotely. Using line-by-line coding, data were analysed using a hybrid approach that incorporated both a deductive, theoretical process and an inductive, data-driven process.

Results Participants included 29 dentists and nine dental nurses from UDCs across England. Twelve themes were identified and grouped into positive and negative experiences. Positive experiences were: role fulfilment and having a sense of purpose; team unity and collective coping strategies; and strategic teamwork and preparedness for effective organisation of care. Negative experiences included: feeling undervalued and frustrated due to fragmented guidance and communication; sense of unfairness generated by relational challenges; patient demand outstripping UDC capacity; complex decision-making; uncertainty over safety; suffocating PPE hindering effective communication; ineffective communication channels across healthcare sectors; lack of commitment to remote video consultations; and variable referral quality.

Conclusions Participants reported experiencing a number of emotional challenges that appeared to be exacerbated by an unsupportive environment, often due to lack of leadership. However, positive experiences and coping strategies were also identified. Collective and sustained efforts at system level to improve the resilience and mental wellbeing of the current and future dental workforce and integration of dentistry into wider healthcare infrastructures are needed.

\section{Introduction}

On 11 March 2020, the World Health Organisation (WHO) declared SARS-CoV-2 and its associated respiratory disease (COVID-19) to

${ }^{1}$ University of Plymouth, Peninsula Dental School, Plymouth, UK; ${ }^{2}$ University of Plymouth, Centre for Health Technology, Plymouth, UK; ${ }^{3}$ University of Plymouth

Peninsula Medical School, Plymouth, UK.

*Correspondence to: Robert Witton

Email address: robert.witton@plymouth.ac.uk

Refereed Paper.

Accepted 22 July 2021

https://doi.org/10.1038/s41415-021-3375-3 be a global pandemic. ${ }^{1}$ To control the spread of the virus, different epidemiological and public health measures were implemented across the globe. In the UK, this involved a national lockdown commencing on 23 March 2020. ${ }^{2}$ Dental services were also disrupted due to the perceived risk from certain aerosol generating procedures (AGPs), which were identified as a potential route of transmission of SARS-CoV-2. ${ }^{3}$ Members of the dental team engaged in clinical dentistry are believed to have high potential exposure risk to SARS-CoV-2 due to their necessary and close proximity with colleagues and patients. ${ }^{4}$
On 25 March 2020, in an attempt to limit transmission of COVID-19, following advice of NHS England, all routine, nonurgent dental care in England ceased and dental practices were asked to establish a remote urgent care service. This involved providing a telephone triage service for patients with urgent needs and provision of advice, analgesics and antimicrobials, where appropriate (AAA). ${ }^{5}$ Urgent Dental Care centres (UDCs) were established across England to provide emergency face-to-face care where clinically necessary. The official standard operating procedure (SOP) for 
UDCs was published by NHS England and NHS Improvement on 15 April, ${ }^{6}$ and UDCs remained the only service providing faceto-face care until the resumption of dental services from 8 June $2020 .^{5}$

Given the uncertainty around the nature of the disease and its routes of transmission, the effectiveness of existing infection prevention and control (IPC) measures in the dental setting were considered inadequate. As a result, enhanced personal protective equipment (PPE) was recommended for direct patient care, including respiratory FFP2/FFP3 masks and gowns for many operative procedures. A British Dental Association survey early in the pandemic showed significant shortcomings in UDC operations, with over half (54\%) of the respondents reporting that PPE shortages were impacting on the operational delivery of UDCs. ${ }^{7}$

Evidence from the wider healthcare literature has shown that during COVID19 , frontline healthcare workers experienced intense emotional turmoil, ${ }^{8}$ heightened levels of anxiety and distress, ${ }^{9,10}$ and poor sleep quality, ${ }^{11}$ increasing their risk of developing mental health and wellbeingrelated problems. ${ }^{12}$ Emerging research has also identified positive emotional responses to working during the pandemic, such as increased sense of team unity, purpose and reward, as well as the adoption of helpful coping mechanisms. ${ }^{911}$ However, research exploring the experiences of frontline dental care professionals during the COVID-19 pandemic has been limited to a handful of quantitative studies. These studies have focused on processes implemented to operationalise UDCs and on their clinical activity and output. ${ }^{13,14,15}$ To date, no published study has explored and reported frontline experiences of what it was actually like to work within a UDC and why. This is surprising considering the long-term impact of COVID-19 and UDC operations on dentistry, the richness of lessons potentially learnt from this time and the high likelihood that processes, such as the ones used during COVID-19, will be required in the future again.

Compared to the type of information retrieved from quantitative measures, qualitative research can provide greater insight and understanding into people's lived experiences and perceptions than can be achieved by quantitative studies. ${ }^{16}$ This study therefore aimed to address the aforementioned knowledge gap by using a qualitative approach to examine frontline staff perspectives and experiences of working (remotely and face to face) within UDCs during COVID-19.

\section{Methods}

Study design and theoretical framework

A qualitative research study adopting a phenomenological approach to explore the lived experiences of dental professionals working at UDCs during the pandemic. ${ }^{17}$

\section{Ethical approval}

Ethical approval was obtained from the Faculty of Health and Human Sciences Research Ethics and Integrity Committee of the University of Plymouth (ref:19/20-1300). All participants consented to participate in the study and to have their data used as part of the research.

\section{Stakeholder engagement}

An NHS Community Dental Service Senior Dental Officer (MBD), with an academic and research background, who currently provides care at a UDC, provided input and advice on the design, research materials and data collection tools incorporated in this study. She also participated in the verification of data, implications and dissemination plans for research.

\section{Participants}

Participants included dentists and dental nurses involved in care delivery (face to face and/or remote) at UDCs in England. Due to logistics, ethical constraints and the urgency for collecting data during the pandemic, dentists or managers solely involved in setting up the UDCs, and dental professionals offering dental care in UDCs in other devolved nations, were not considered for participation. In the first instance, participants were recruited through professional networks using purposive sampling and snowballing thereafter. ${ }^{18,19,20}$ To ensure a diverse sample, stratification was also used. ${ }^{18,21}$

\section{Data collection}

Semi-structured interviews lasting 45-60 minutes, using a pilot-tested topic interview guide, were conducted by a dentist with extensive research experience, who was not involved in UDC care provision (AP). The interview guide, available in the online supplementary information, was informed by the findings of peer-reviewed and grey literature reports related to dental care provision during the COVID-19 pandemic. The interviews took place between June and August 2020 using Voice over Internet Protocol technologies (Zoom, Skype) or telephone (as per the preference of the interviewee). Field notes were made shortly after the interviews were completed. Interviews were digitally audio-recorded and transcribed verbatim (AP). Transcripts were anonymised and re-read for accuracy, enabling the researchers to immerse themselves within the data. Anonymised transcripts were then uploaded onto NVivo software (version 12) for coding and management.

\section{Data analysis}

Using line-by-line coding, data were analysed using a hybrid approach that incorporated both a deductive, theoretical process and an inductive, data-driven process (RB). ${ }^{18,22,23}$ The former produced a set of a priori codes/topic themes derived from the research aims and the interview guide/schedule that was used in the study. The latter approach produced a series of data-driven post-empirical codes and subthemes that derived from the examination of data generated from the interviews.

Independent coding by more than one researcher of a random subset of interviews, sense checking processes and team discussions provided opportunities for coding decisions to be challenged and adjusted (RB, AP, HW, MP, MBD), ensuring a high degree of inter-rater reliability. Once coding was completed, emergent themes and subthemes were discussed and refined during 'analysis' team meetings (AP, MP, HW). One member of the data analysis team (MBD) was involved in treatment of patients in UDCs during the pandemic. Three out of five team members involved in data analysis were not clinicians, thus allowing diverse perspectives and interpretations in the interpretation of data. Consideration was given on how the team member characteristics and experiences may have influenced the analysis.

\section{Results}

Thirty-eight frontline staff (29 dentists and nine dental nurses [DNs]) working in UDCs providing face-to-face and/or remote care in different settings including community, hospital and general dental practice across 
England were recruited. The demographic characteristics of our sample are presented in greater detail in Table 1. Our sample included a diverse range of dentists, such as salaried community dentists (CDS), dental core trainees (DCTs) and general dental practitioners (GDPs). Among our participants, three dentists were specialists, with one working solely in a teaching dental hospital, one in community as well as in an oral surgery hospital department, and one as a practice owner of a specialist referral practice.

Our thematic analysis identified 12 themes. We grouped these themes into positive and negative frontline experiences, to improve accessibility to the reader. Positive experiences were: role fulfilment and having a sense of purpose; team unity and collective coping strategies; and strategic teamwork and preparedness for effective organisation of care. Negative experiences included: feeling undervalued and frustrated due to fragmented guidance and communication; sense of unfairness generated by relational challenges; patient demand outstripping UDC capacity; complex decision-making; uncertainty over safety; suffocating PPE hindering effective communication; ineffective communication channels across healthcare sectors; lack of commitment to remote video consultations; and variable referral quality. A description of each theme accompanied by participants' quotes is provided in Table 2.

\section{Positive frontline experiences}

\section{Role fulfilment and having a sense of} purpose

For many participants, being able to help patients 'in a time of crisis, when people need you the most' (CDS7) was a beneficial and valued experience and was perceived as something 'quite special' (DN9). Benefits of being able to help also appeared multifaceted, contributing to personal and professional satisfaction and fulfilment. For example, 'the benefit obviously was my ability to contribute to the wider NHS and be able to help patients and take them out of pain and the professional satisfaction of doing the right thing' (GDP 4).

Having a sense of 'purpose' (CDS3) and 'a little bit of normality' (CDS1) was also repeatedly identified as beneficial. For some participants, this was perceived as a vital component of maintaining their own health and wellbeing, as well as an opportunity for 'social interaction' (GDP3) during the most stringent lockdown period. Combining a

Table 1 Demographic characteristics

\begin{tabular}{|c|c|c|}
\hline Characteristics & \multicolumn{2}{|l|}{ Results } \\
\hline \multirow{2}{*}{ Gender } & \multicolumn{2}{|l|}{ Male: $n=12(31.6 \%)$} \\
\hline & \multicolumn{2}{|l|}{ Female: $n=25(68.4 \%)$} \\
\hline \multirow{2}{*}{ Years of experience } & Dentists & Mean 17.16 years (SD 10.89; range $2-38$ ) \\
\hline & Dental nurses & Mean 23.12 years (SD 11.45; range $7-41$ ) \\
\hline \multirow{7}{*}{ Role } & \multirow{4}{*}{ Dentists: $n=29(76.3 \%)$} & General dental practitioners: ${ }^{*} n=13(44.8 \%)$ \\
\hline & & Community dentists: $n=11(38.0 \%)$ \\
\hline & & Specialist dentists: $n=3(10.3 \%)$ \\
\hline & & Dental core trainees: $n=2(6.9 \%)$ \\
\hline & \multirow{3}{*}{ Dental nurses: $n=9(23.7 \%)$} & Community dental nurses: $n=5(55.6 \%)$ \\
\hline & & General practice dental nurses: ${ }^{* *} n=2(22.2 \%)$ \\
\hline & & Hospital dental nurses: $n=2(22.2 \%)$ \\
\hline \multirow{10}{*}{ Region } & \multicolumn{2}{|l|}{ East Midlands: $n=3(7.9 \%)$} \\
\hline & \multicolumn{2}{|l|}{ East of England: $n=1(2.7 \%)$} \\
\hline & \multicolumn{2}{|l|}{ London: $\mathrm{n}=1(2.7)$} \\
\hline & \multicolumn{2}{|c|}{ Midlands (East and West): $n=1(2.7 \%)$} \\
\hline & \multicolumn{2}{|l|}{ North East: $n=2(5.2 \%)$} \\
\hline & \multicolumn{2}{|l|}{ North West: $n=6(15.7 \%)$} \\
\hline & \multicolumn{2}{|l|}{ South East $n=9(23.6 \%)$} \\
\hline & \multicolumn{2}{|l|}{ South West: $n=12(31.6 \%)$} \\
\hline & \multicolumn{2}{|l|}{ West Midlands: $\mathrm{n}=1(2.7 \%)$} \\
\hline & \multicolumn{2}{|c|}{ Yorkshire and the Humber: $n=2(5.2 \%)$} \\
\hline \multirow{4}{*}{ UDC setting } & \multicolumn{2}{|c|}{ Community service: $n=18(47.4 \%)$} \\
\hline & \multicolumn{2}{|c|}{ General dental practice: $n=17(44.7 \%)$} \\
\hline & \multicolumn{2}{|l|}{ Hospital service: $n=2(5.2 \%)$} \\
\hline & \multicolumn{2}{|c|}{ Community and hospital service: $n=1(2.7 \%)$} \\
\hline
\end{tabular}

sense of purpose and being able to help, some participants also expressed a 'sense of pride' (GDP13) when reflecting on their UDC experience and related accomplishments. This sense of pride was related to the number of patients they had helped, the absence of their own patients needing UDC attention and level of teamwork achieved.

Patient gratitude and regular positive patient feedback was also identified by participants as a positive and, at times, unusual experience. Patients' positive feedback often became a source of motivation and empowerment for UDC staff. However, participants also reported that patient gratitude was gradually diminishing as patient expectations increased following the resumption of general dental services.
Team unity and collective coping strategies for resilience

Participants reported that working in UDCs strengthened working relationships, brought team members closer and helped them to appreciate each other more. It also gave some participants the opportunity to work with colleagues from other professional networks and share learning. Enhanced teamwork was uniformly acknowledged as a positive and 'refreshing" (SD2) experience, with participants reporting that what stood out was 'how well everybody pulled together' (GDP5). However, for some, team morale was believed to be wavering given the unexpected, extended duration of UDC operation.

A number of key coping strategies that fostered team unity and facilitated resilience 
Table 2 Theme and subtheme description and supporting quotes (cont. on page 4)

\begin{tabular}{|c|c|c|}
\hline Theme description & Subthemes & Supporting quotes \\
\hline \multicolumn{3}{|l|}{ Positive frontline experiences } \\
\hline $\begin{array}{l}\text { Role fulfilment and having a sense of purpose } \\
\text { Participants reported that helping patients and } \\
\text { having a sense of purpose led to personal and } \\
\text { professional fulfilment }\end{array}$ & $\begin{array}{l}\text { - Sense of normality } \\
\text { - Ability to help } \\
\text { - Sense of pride } \\
\text { - Patient gratitude }\end{array}$ & $\begin{array}{l}\text { 'The benefit obviously was it was my ability to do to contribute to the wider NHS } \\
\text { and be able to help patients and take them out of pain and the professional } \\
\text { satisfaction of doing the right thing' (GDP4) } \\
\text { 'Staff could see the gratitude of patients that we were getting out of pain and they } \\
\text { felt empowered by it...' (GDP8) }\end{array}$ \\
\hline $\begin{array}{l}\text { Team unity and collective coping strategies } \\
\text { Participants reported that teamwork and team } \\
\text { unity contributed to positive work experiences. They } \\
\text { described coping strategies they adopted to improve } \\
\text { their resilience and mental wellbeing }\end{array}$ & $\begin{array}{l}\text { - Teamwork and unity } \\
\text { - Huddles } \\
\text { - Coping strategies }\end{array}$ & $\begin{array}{l}\text { 'I think there's been a real effort of really good teamwork [...] that's stuck with me. } \\
\text { That stood out, how well everybody pulled together' (GDP5) } \\
\text { 'I think it has brought us a lot closer together [...] everybody's very much been } \\
\text { looking out for each other and making sure that everybody's happy with what's } \\
\text { been going on' (GDP13) }\end{array}$ \\
\hline $\begin{array}{l}\text { Strategic teamwork and preparedness for } \\
\text { effective organisation of care } \\
\text { Strategies described by participants to optimise } \\
\text { workforce availability and organisation of care in } \\
\text { order to ensure efficient UDC care delivery }\end{array}$ & $\begin{array}{l}\text { - Challenges in workforce } \\
\text { availability } \\
\text { - Strategic team } \\
\text { composition } \\
\text { - Practice organisation } \\
\text { - Procedural preparations }\end{array}$ & $\begin{array}{l}\text { 'If you have an AGP procedure, we have an extra surgery now where we can doff } \\
\text { and use that extra surgery to carry on, so we don't have to have an hour not doing } \\
\text { anything and waiting around. So that's much more efficient' (CDS7) } \\
\text { 'You always have to plan completely in advance. You can no longer send someone } \\
\text { in and out [...] You have to think about who comes in, who goes out, how you fill } \\
\text { the room, you are all PPEd [sic] up [...] you have to prepare much better in advance' } \\
\text { (GDP7) }\end{array}$ \\
\hline
\end{tabular}

Negative frontline experiences

Feeling undervalued and frustrated due to fragmented guidance and communication Participants described that they felt undervalued and that their service was not shown any appreciation. These feelings stemmed from poor communication and support from governing bodies

\section{Sense of unfairness generated by relational challenges \\ Participants expressed resentment towards people on furlough, staff who believe they had failed their fit tests and non-UDC dentists who did not support the UDC service}

- Slow national communication

- Fragmented guidance

- Lack of support

- Lack of recognition of own efforts and dentistry as a profession

- Attitudes towards people on furlough

- Overworked but same reimbursement

- Lack of support by local practitioners

\section{Patient demand outstripping UDC capacity}

The patient demand was overwhelming and was outstripping UDC capacity, which heightened the stressfulness of UDC environments. The efficiency of the UDC service was negatively affected by availability and cost of PPE, fit testing, availability of UDCs, referral systems and paperwork
- High patient demand

- High workload

- Restricted PPE availability

- Slow fit testing

- Limited UDC availability

- Destructive paperwork

- Altered prescribing behaviour

'The general feeling is one of disappointment from the GDC where they sort of left us in the lurch [...] it was poor form from our governing bodies and people that regulate us. They did not seem to really support dentists' (CDS1)

'Nationally, I think things could have been done better, especially from the CDO's office, there were a lot of confusing messages coming out...' (GDP13)

'The big barriers really are things like the complete lack of communication and coordination nationally. I think it created a confusing environment' (GDP7)

'Some of the nurses couldn't wear FFP3 and then the more work came over to the nurses, which could wear it. And after a while, we just started being tired. And obviously those nurses couldn't do basically any jobs [...] me and the other nurses are saying this is not fair because we've been working like this for months. This particular nurse she didn't work at all, she got the same money...' (DN5)

'It's created a little bit of resentment within some people that are working [...] everyone else is off and we're coming in every day and working harder than normal [...] other people are still off enjoying the sunshine, sitting in the garden' (CDS2)

'The volumes were skyrocket high [...] the daily volumes in some cases were 1,600, 1,700 calls a day for the whole of [named city] which is very high' (GDP7) II just think that the sheer volume of patients, especially when GDPs were closed, was just enormous. It was just overwhelming, absolutely overwhelming' (CDS6) 'I don't think the referral system has a function to stop overflow. So some patients were referred to like [named area] and they might have to wait five days to be seen, whereas we don't get any referrals in [named area]' (GDP11)

'Unfortunately, I felt we were like almost forced to prescribe more antibiotics than we needed to because, only because of the current situation, especially initially' (GDP4)

'It took almost a month to actually get our PPE [...] we were ready to go after a week and we were just sitting ducks because we had no PPE' (GDP1)

'Fit testing, it has been so slow [...] and let's face it, this can only compromise patient care' (GDP11)

\section{Complex decision-making}

Participants described how the complex decisions they had to make about patient care negatively
- Emotional demands in decision-making

- Irreversible decisions impacted on their experiences

'We were hearing about colleagues who were not sleeping because of whether they made the right decision for a patient you know, they were overwhelmed with the decision-making, the number and complexity of the decisions' (CDS5)

'I've never spoken to people who were so desperate with pain and that was quite stressful. There was one occasion, I just came off the phone and cried because it just felt that there were people out there and you couldn't do anything to help them [...] it was distressing...' (CDS8)

\section{Uncertainty over safety}

Participants expressed fears for their safety, patients' safety, and the health and safety of their family. They linked these fears with the appropriateness of PPE

\section{Suffocating PPE hindering effective} communication

The physicality of wearing PPE left participants feeling suffocated. PPE acted as a physical barrier for effective communication

\section{- Personal protection}

- Patient protection

- Health of family

- Suffocating PPE

- Physical barrier in communication
'To start with the anxiety levels were massive, particularly, you know, does my mask fit me properly? Is it actually going to protect me?' (DS1)

'My ultimate worry was not being protected, moving the virus around, you know, I've got a family, I've got a mum who was shielding at the time [...] it was a very, very stressful time' (DN9)

'When it was very hot, I felt very breathless in it, and I was kind of trying to get the patient out quick so I could take the mask off. Which maybe made me rush through the post-op instructions potentially' (GDP2)

'That was challenging in the way that lots of people, they read your lips to understand what you are saying...' (CDS6) 
Table 2 Theme and subtheme description and supporting quotes (cont. from page 3)

\begin{tabular}{|c|c|c|}
\hline Theme description & Subthemes & Supporting quotes \\
\hline \multicolumn{3}{|l|}{ Negative frontline experiences (cont.) } \\
\hline $\begin{array}{l}\text { Ineffective communication channels across } \\
\text { healthcare sectors } \\
\text { Communication with other healthcare providers } \\
\text { was hindered by the lack of shared access to patient } \\
\text { medical details. Remote prescribing introduced new } \\
\text { challenges to UDC dental teams }\end{array}$ & $\begin{array}{l}\text { - Challenges in } \\
\text { communication with other } \\
\text { healthcare providers } \\
\text { - Challenges in remote } \\
\text { prescribing }\end{array}$ & $\begin{array}{l}\text { 'I think we're at a big disadvantage where we were not able to access the bloods, } \\
\text { we had to ask the doctors to send us the medical history, send us blood results. So } \\
\text { as we were not part of their system and I felt that we were left out. And we needed } \\
\text { that information, but trying to get through to the doctors was very difficult' (CDS2) } \\
\text { 'On the one side, there was a push from commissioners about remote prescribing, } \\
\text { on the other side, the regulation doesn't add up and nor do the systems add up. So } \\
\text { there were, there was an expectation that prescribing would happen remotely and } \\
\text { that would be by creating prescriptions, scanning it and sending it, the whole thing } \\
\text { was broken' (GDP7) }\end{array}$ \\
\hline $\begin{array}{l}\text { Lack of commitment to remote video } \\
\text { consultations } \\
\text { There was limited uptake of remote video } \\
\text { consultation opportunities. Participants who did not } \\
\text { engage with video consultations perceived them as } \\
\text { unnecessary, time-consuming or inappropriate for } \\
\text { certain patient groups. Some benefits of their use } \\
\text { were identified }\end{array}$ & $\begin{array}{l}\text { - Creaky computer systems } \\
\text { - Perceptions about patient } \\
\text { ability to engage } \\
\text { - Perceived challenges } \\
\text { - Benefits of teledentistry }\end{array}$ & $\begin{array}{l}\text { 'But I wonder how long the } 30 \text { calls which I received on a Monday morning would } \\
\text { have taken me if I was doing a video consultation as opposed to on the telephone' } \\
\text { (DCT2) } \\
\text { 'It was good for assessing facial swelling but not diagnosing anything intraorally } \\
\text { because the camera, with patients trying to get them to hold the camera, the right } \\
\text { position is very difficult to see exactly what was going on in the mouth, it was very } \\
\text { jumpy' (CDS2) }\end{array}$ \\
\hline $\begin{array}{l}\text { Variable referral quality } \\
\text { Referrals the participants received and processed were } \\
\text { of variable quality. There was a surge of inappropriate } \\
\text { AGP referrals when the practices first opened. The } \\
\text { quality and completeness of referrals, however, } \\
\text { improved after the resumption of dental services }\end{array}$ & $\begin{array}{l}\text { - Hit or miss referral quality } \\
\text { - Inappropriate referrals } \\
\text { - Lack of radiographs } \\
\text { - Challenges referring } \\
\text { unregistered patients }\end{array}$ & $\begin{array}{l}\text { 'As always, there are some dentists that manage very well and some that seem to } \\
\text { try and cut corners and refer everything' (CDS1) } \\
\text { 'One thing I did realise quite quickly with this whole project, with this sort of urgent } \\
\text { dental centres is that dentists as a profession, we refer terribly. Our referral is } \\
\text { absolutely abysmal' (GDP4) } \\
\text { 'When we started doing AGPs as a direct referral, I did find that there was } \\
\text { immediately an increase in inappropriate referrals [...] we were getting some referrals } \\
\text { for AGP that I think were a sort of a bit of a dumping ground, to be honest' (GDP6) }\end{array}$ \\
\hline
\end{tabular}

were reported. These included: morning huddles and debriefing sessions, which provided an opportunity for 'checking in on each other' (DN8); using an 'anxiety scale' (DN6) to monitor mental health and wellbeing and to support team members in need; using a dedicated 'wobble room' (DN9) for people to unwind when they felt overwhelmed; ensuring 'enough breaks' (GDP11); not working out of hours; spending time outdoors during breaks and offering relaxation activities such as 'colouring' (DN9) and 'meditation' (SD2). Participants highlighted the need to sustain the efforts to maintain and improve teams' mental health and wellbeing beyond the pandemic, and that changes at system level and working culture are required to facilitate these efforts in the long term.

\section{Strategic teamwork and preparedness for effective organisation of care}

UDC teams employed different strategies to maximise the efficiency of the service; for example, when staffing issues arose as a result of staff being redeployed, shielding, contracting COVID-19 or being furloughed. Strategic decisions that optimised teams' organisation included: a) splitting the team into smaller teams, which worked on different days/sessions; b) splitting clinicians' workload and responsibilities (that is, clinical vs triage role), either by session or day rotations to allow a break from PPE; c) providing continuity of care by dentists treating patients they had triaged, or as per their area of interest or expertise (for example, paediatrics etc); and d) allocating triaging responsibilities to team members who were shielding, or were uncomfortable taking an 'active clinical role' (CDS9), due to increased risk of COVID-19 complications. Having a mixture of expertise within the team, availability of clinical support, working in smaller and familiar teams, having clearly defined roles, and operating in a supportive and open team culture were identified as important for a more efficient UDC operation. These findings indicate the importance of good leadership and management, and teams which are innovative, flexible, adaptable and willing to embrace change.

In some community and general dental practice UDC settings, a decision was made for 'two dentists working with one patient' and a runner nurse 'cleaning and bringing the equipment' (SD2). This enabled dentists to support each other, share expertise and ultimately enhance their 'confidence' (CDS1). This decision was also driven by the fact that there was ' $a$ lot of oral surgery at the beginning' and participants 'were conscious that the buck stops with them' (CDS1). Interestingly, having 'oral surgeons in the hub' was described as ' $a$ huge bonus' (GDP1) and this was reiterated by the majority of the participants.
To ensure UDCs were COVID-19 secure, changes in the practice layout were often required. Having 'enough surgeries' (CDS4) and sufficient clinical space greatly facilitated an appropriate response to COVID-19 restrictions. When clinical space was limited, some participants used the fallow time to 'triage patients' (CDS4). Staggering appointments helped to maintain service efficiency, while minimising the risk of infection transmission introduced by staff or patient crossover. To maximise efficiency, some participants also reported planning AGP appointments before lunch or the final afternoon session in order to limit the detrimental impact of fallow time on appointment availability.

The level of preparation required because of COVID-19 related restrictions was repeatedly acknowledged by participants; 'you always have to plan completely in advance' (GDP7), especially for AGP procedures. The importance of holding morning huddles and debriefs to facilitate preparedness, quality improvement, catch up with changing guidelines and maximise UDC efficiency was highlighted by most participants. Holding such team meetings were not common practice before the pandemic but were now considered 'incredibly helpful' (DN4), suggesting a positive change moving forwards.

Dental nurses and runner nurses were recognised as key members of the team, and 
their role was seen as integral in the efficient functioning of the UDCs. This was particularly important in terms of procedural preparation, including IPC, ensuring all necessary instruments and equipment were available when required. The importance of nurses was echoed by the vast majority of participants 'the nurses, there are some really extraordinarily hardworking compassionate people' (CDS8).

\section{Negative frontline experiences}

Feeling undervalued and frustrated due to fragmented guidance and communication

Some participants felt undervalued, ignored and opined that they had been 'left in the lurch' (CDS2) by the regulatory and national governing bodies. Delayed response, the inconsistent and 'ever-changing guidelines' (GDP4), and lack of clear communication at national and senior management level regarding remuneration and UDC operations were identified as particularly stressful and left participants feeling unsupported. One of the greatest barriers to the timely operation of UDCs, identified unanimously by participants, was the 'bureaucracy' involved (CDS10) and 'complete lack of communication and coordination nationally' (GDP7), particularly early on in the pandemic. Particular dissatisfaction was expressed with the level of communication from the Office of the Chief Dental Officer for England (OCDO), especially when compared to the other devolved nations. However, participants acknowledged that communication from the OCDO improved during the course of the pandemic. A lack of consideration, assurance and communication regarding increased costs associated with operating as a UDC was a concern identified by the majority of participants, with many feeling undervalued or 'taken advantage of (GDP11) as a result.

Faced with a lack of nationally agreed SOPs and guidance, practice and service managers initially had to resort to other channels of communication to develop their own SOPs, such as other UDC providers, dental public health consultants and local commissioners. In contrast to national communication, communication both within and between local networks and providers was praised by the majority of participants. The importance of Local Dental Committees (LDCs) in disseminating new pieces of information and keeping 'things cohesive between different practices' (DCT2) was also emphasised by a number of participants.
The inadequacy of communication had important implications for how those involved in UDCs perceived the value attributed to dentistry by national bodies and the government. This was more strongly voiced when discussing how the resumption of general dental services had been communicated to the profession on 29 May 2020. Frustration was expressed around the timing and the method of the announcement: 'we should not hear from the BBC that practices are opening at the same time as the damn public... We should know beforehand' (CDS11). Interviewees' responses suggested that COVID-19-related perceptions of being undervalued were reinforcing previously held beliefs about dentistry being treated as inferior in comparison to other healthcare services: 'It's [dentistry] always the forgotten black sheep of the family' (GDP3).

\section{Ineffective communication channels across}

healthcare sectors

Participants reported difficulties in communicating with other healthcare providers such as GPs and hospital consultants, often attributing this to a lack of 'transferability of information' (GDP7). Participants regularly acknowledged the complexity of triaging patients with complex medical needs and the additional 'legwork' (CDS7) this required. Dentists' lack of access to patients' electronic medical records was seen as ' $a$ big disadvantage' (CDS2), as it meant UDC staff often had to contact medical colleagues to obtain information crucial for patient management, especially when patients were unable to provide details of their diagnosis and/or medication.

In addition to accessing medical health records, remote prescribing was also described by participants as 'a big barrier' (SD3) or 'nightmare' (SD3) in delivering efficient and effective care. Different UDCs dealt with remote prescribing in various ways, such as sending 'prescriptions out to patients' (DCT1), asking patients to 'collect the prescriptions' (GDP4), faxing the prescription to the pharmacist and, more often than not, contacting the pharmacist, sending the prescription electronically via e-mail and then posting a paper copy. Although commonly encountered, this last practice was described as particularly problematic and caused further delays in patient care: 'That was, again, relying on getting hold of the pharmacy, that was timeconsuming. Some of the e-mail addresses they gave weren't actually correct. So, I had a patient wait over a week for prescription' (CDS5). Having a list of available pharmacists per locality was seen as a helpful way to overcome prescribing difficulties outlined above.

\section{Sense of unfairness generated by relational} challenges

Relational difficulties underpinned by a sense of unfairness were experienced by several participants, which sometimes affected team morale. Reported experiences of staff intentionally failing mask fit testing or refusing to engage in clinical face-to-face roles due to their perception of risk led to some participants reporting a perception that they had been taken advantage of due to the impact it had on their workload. Participants also described feelings of disappointment towards the NHS with regards to poor availability of and access to PPE and fit testers: 'NHS England kept on saying "there's a drop of PPE to arrive". We never saw any of it' (GDP 3).

Another reported area of contention was interviewees' perception that people who were on furlough were 'enjoying the sunshine, sitting in the garden' (CDS2) 'and still being paid' (SD1), in contrast to the UDC staff who worked throughout the lockdown. Some participants also expressed frustrations with the lack of support shown from fellow colleagues who ignored 'their duty of care' (GDP11) and tried to 'pass the buck' (GDP6) to the UDCs. For example, it was reported that some practices were not available to triage their own patients, did not resume their dental services in a timely manner after 8 June, or did not offer AGP treatments. Notably, LDCs addressed some of the above issues and consequently 'made things cohesive between different practices' (DCT2). The announcement by the Chief Dental Officer regarding abatement and $20 \%$ targets was perceived by some as 'an excuse for inactivity for practitioners' (GDP8). Some participants suggested that the NHS should have asked NHS practitioners to work in UDCs or taken a more punitive approach towards the 'notorious offenders' (GDP1).

\section{Patient demand outstripping UDC capacity}

Most participants reported that patient demand dramatically exceeded UDC capacity, describing the daily volume of patients requiring care (either remote or face to face) as 'skyrocket high' (GDP7). 
One participant vividly described working in a UDC as working in a 'walk-in centre on steroids' (SD3). Feeling tired or exhausted as a result of the unique challenges they faced and substantial demands placed on them when working within a UDC were frequently reported. To try and meet increased service demand, dental teams often had to work additional hours, sometimes 'without any breaks' (CDS8), exacerbating existing feelings of exhaustion and fatigue. For example, 'it was quite intense and tiring because it's not what our roles generally are and not what we're used to' (CDS2). Balancing childcare responsibilities with a heavy UDC clinical workload was also reported as a factor contributing to anxiety and stress.

Some participants expressed dissatisfaction with how the level of demand placed on individual UDCs varied substantially. For example, they reported poor UDC availability across certain geographical areas, as well as an unequal overflow and distribution of referrals among UDCs which they attributed to inadequacies in IT referral systems and algorithms.

COVID-19-related restrictions of social distancing, additional time required for cleaning and disinfecting the surgeries, and fallow time further lowered UDC capacity. Availability and costs of PPE and challenges in accessing fit testers had a direct impact on clinical capacity of UDCs. PPE access and availability was described as 'the biggest barrier to providing care' (GDP5). The amount of paperwork required to evidence UDC activity was viewed as a task that generated 'duplication' (GDP3) and distraction for clinical staff, thus reducing clinical output - 'they keep putting more paperwork, more paperwork. It feels like actually, we're here, we should be doing dentistry' (CDS10).

Furthermore, the perceived lack of support from general dental practice colleagues in complying with AAA protocols, referring appropriately and delaying the resumption of their services in a timely fashion was reported as a factor which exacerbated the UDCs' inability to meet the high patient demand. Participants frequently acknowledged that it was patients who had 'really suffered' (CDS11) due to the restricted UDCs' capacity and closure of dental services. As a result, some participants felt 'forced' (SD1) to prescribe antibiotics and change their attitudes towards prescribing in order to help patients who could not be seen face to face.

\section{Complex decision-making}

The complexity of cases that UDCs dealt with, especially in regard to underlying medical conditions such as 'patients who were on dialysis, undergoing heart valve transplants, patients who are on immunosuppressants, undergoing chemotherapy' (CDS2), was described by participants as particularly challenging. Compounded by lack of expertise, participants often reported pushing themselves to their 'absolute limit' (CDS5). Due to the nature of the UDC service, the high number and complexity of often irreversible decisions UDC staff had to make left some participants feeling vulnerable to litigation: 'we're waiting for the first solicitor's letter saying you didn't open up that tooth, you took it out...' (SD3). Participants also described the emotional and mental demands endured in making decisions when triaging or treating patients they had not met before: 'by the time you've seen five [patients], you're shattered, because they're five completely new patients you don't know anything about' (CDS3). A sense of 'guilt' (GDP3) about not being able to help all patients due to limited UDC capacity and having to prioritise emergency care was also often reported.

\section{Uncertainty over safety}

High levels of anxiety were reported around personal health and protection: 'to start with, the anxiety levels were massive, particularly, you know, does my mask fit me properly? Is it actually going to protect me?' (SD1). A fear 'of the unknown' (DN6) appeared particularly prevalent at the beginning of the pandemic. In response to these fears, some participants decided to take precautionary actions such as taking out a life insurance policy. Anxiety levels appeared particularly elevated for participants at high risk of severe COVID-19 complications and/or those with young/elderly family members.

The fear of contracting the virus, or passing it on to colleagues, patients and/or loved ones, was associated with the perceived level of protection provided by the available PPE. These worries and anxieties were heightened for some participants who received 'counterfeit PPE' (GDP7) or 'expired masks' (CDS4) and felt they had to 'carry on seeing patients with expired masks' (CDS4), whose elastic bands kept 'snapping' due to degradation (GDP1). However, the stringent IPC protocols followed ensured a general sense of personal and patient safety among participants: 'I feel safer going to work in a hot site than I do going to a shop'(SD1).
Interestingly, a small number of participants perceived some of the additional measures and PPE (that is, head and foot covers) as an 'expensive overkill' (DN7), especially for nonAGP procedures.

\section{Suffocating PPE hindering effective communication}

Wearing level three PPE was seen as 'very challenging to work in' (CDS5), with participants describing it as 'really uncomfortable' (DN2), 'unpleasant' (GDP3), 'exhausting' (SD1) and 'suffocating' (GDP2). Sessional use of enhanced PPE left some participants feeling 'breathless' (GDP2), while others failed to stay hydrated: 'obviously you didn't drink enough [...] some of the dental nurses, most of them, even now, we have urine infections' (DN5). These difficulties were magnified by the unusually hot weather in the UK during the first lockdown and the lack of air conditioning: 'I was, sweating buckets, really, really hot' (CDS5). Such experiences led some participants to spend less time with patients than they would have liked to. Linked to increased PPE use, participants also described practical challenges of managing clinical waste, which saw a 'fivefold increase' (GDP7), while raising concerns over the amount of plastic discarded and environmental impacts.

PPE was seen as 'a physical barrier' (CDS7) hindering effective clinician-patient communication. Loss of the 'personal touch' (DN2) and the removal of other forms of non-verbal communication such as facial expressions and body language was perceived as a barrier, especially in terms of restricting their ability to provide reassurance to nervous patients. Reported difficulties in communication, as a result of PPE, appeared more evident 'when treating children, adults that have difficulties with hearing or patients with dementia' (CDS7) and people with learning difficulties. In addition, PPE was seen as being intimidating for patients, especially children and patients with additional needs. Difficulties in effective communication were also reported between team members in the surgery. Participants attributed communication difficulties mainly to the inability to lip-read and read facial expressions. Use of 'flashcards' (DN8) was utilised by one UDC to overcome some of the dentist-patient communication issues encountered. Other strategies used to put patients at ease included: using descriptive 
terms during triage, such as 'stormtroopers or beekeepers' (CDS7); signposting to 'leaflets or websites' with pictures of the staff 'in full $P P E$ '(SD1); and putting 'smiley stickers on the masks' (CDS7) or wearing badges with face pictures on them.

\section{Lack of commitment to remote video consultations}

Some participants engaged with remote consultations via video technology. The most frequently reported barrier affecting the use of video conferencing was the lack of appropriate devices and required infrastructure, with some participants referring to existing computer systems used as 'creaky' (GDP13). In many cases, participants positively described using pictures in e-mails to facilitate patient care. However, it is unclear whether participants were using pictures and e-mails in response to device/infrastructure limitations or a lack of perceived need for video consultations.

Concerns over cost, and the increased length of consultations if video conferencing had been used, were also raised by participants. Worries were expressed that some patients, especially older patients, may not own a smartphone or electronic device, nor have the required digital skills and capabilities. This appeared to discourage some UDC services from engaging with video conferencing.

Reported benefits of using video conferencing technology included providing patient reassurance, especially for those patients who were shielding, too scared to attend, or were being assessed for facial swellings, therefore preventing unnecessary prescription of antibiotics. The use of video, however, was deemed less useful for intraoral assessment.

\section{Variable referral quality}

The majority of participants reported widespread variability in the quality and appropriateness of referrals received by fellow practitioners, with some describing referrals as a 'hit or miss' (CDS7). A lack of information regarding what AAA measures had already been taken often led to referrals being rejected, or the UDC staff carrying out a secondary triage before accepting patients for urgent care as they didn't 'take the GDP's triage at face value' (CDS9). Another reported consequence of poor referral quality, including a lack of 'historical $x$-rays' (GDP1), was the misallocation and waste of clinical time. The introduction of electronic referral systems requiring specific information to be included in the referral ensured that acceptable standards of referrals were met, but was also seen as a barrier when referring unregistered patients. Participants, however, reported that since dental practices were allowed to open, availability of radiographs in referrals improved but the number of inappropriate referrals for non-urgent cases requiring AGPs increased.

\section{Discussion}

This is the first qualitative interview study that explores the frontline experiences and perceptions of dental care professionals working at UDCs during the COVID-19 pandemic. Participants' reported experiences indicate that working within UDCs often generated multifactorial stress and anxiety, particularly when there were concerns over safety and operational challenges. Poor communication and inconsistent advice across various levels of dentistry appears to have consistently contributed to a sense of uncertainty and a perception that governing bodies and senior management had a lack of understanding of, and appreciation for, the work undertaken with UDCs. At the same time, some positive outcomes were also identified, including greater team unity and a strong sense of professional responsibility.

Enhanced teamwork, feeling appreciated and being able to help emerged as important sources of support, corroborating findings from other studies on frontline healthcare and dental staff during the COVID-19 pandemic in the UK. ${ }^{9,11,24,25}$ These positive experiences could be attributed to the continued sense of normality and ability to fulfil their professional roles. In the present study, team unity, especially in terms of 'pulling together', appeared to enhance resilience to the stressful elements of working in a pandemic. Pre-COVID-19, dentistry was already recognised as a stressful profession, with UK dentists reporting high levels of occupational stress (55\%), burnout (86\%) and psychological distress (68\%). ${ }^{26}$ In our study, concerns over personal and family safety, availability of PPE and changing guidelines were reported as common sources of stress. These findings are consistent with those reported by existing studies from the dental and wider healthcare. ${ }^{8,9,10,11,24,27,28}$ Anxiety and distress over rapidly changing guidelines and PPE availability can have a tangible impact on efforts to maintain a sustainable workforce. ${ }^{9}$ The overwhelming demand and reduced capacity provided by UDC services, which is in line with previously reportedly UDC service evaluation data, ${ }^{14,15}$ was highlighted by the majority of our participants and further heightened the stressfulness of UDC environments. Feeling undervalued, fuelled by a lack of communication from governing bodies, was unanimously stated and echoes feelings of 'institutional betrayal' expressed by the dental profession as reported by Collin et al. ${ }^{29}$

Lack of understanding and support at national level and from senior management may have driven enhanced teamwork at a clinic level, as clinicians developed their own support systems. However, this is unlikely to be sustainable and a continued lack of supportive leadership could result in further demoralisation of the workforce. Considering the tangible impact that this pandemic has brought on mental wellbeing, ${ }^{30}$ it is important, now more than ever, that appropriate measures are put in place to support dental teams. It is imperative we use the pandemic as an opportunity to put mental wellbeing awareness at the centre of the dental workplace and education, so that dental professionals and dental teams feel empowered to thrive and patient safety is optimised. ${ }^{31}$

This research identified some helpful coping strategies deployed by staff during the COVID19 pandemic: mindfulness activities, morning huddles and debriefing sessions. Adoption of such strategies within dental practice on a wider scale could have significant benefits for staff and patients. However, it should be acknowledged that there is currently a paucity of evidence in relation to effective mental wellbeing interventions in dental settings ${ }^{32}$ and further research into the effectiveness of such interventions is warranted.

Frontline experiences identified in this study can be used to inform future guidance and action to support health and wellbeing within the dental profession for the benefit of patient care. Collective and sustained efforts at system, organisation and practice level to support the mental health and wellbeing of the UK dental workforce are timely. The recently published 'Mental health wellness in dentistry' framework provides a unique opportunity to make mental health a key priority and to encourage early recognition, intervention and safe signposting among all dental care professionals. ${ }^{31}$ 
Limited UDC capacity left some participants feeling guilty for being unable to help patients. Some participants, as a result, felt forced to change their prescribing behaviour, especially in the beginning of the pandemic, in an attempt to help patients in despair who could not access face-to-face care. This observation is in line with recent data suggesting a $25 \%$ increase in dental antibiotic prescribing between April and July 2020 compared to the same period in $2019 .{ }^{33}$ These findings, combined with the current dental access issue, ${ }^{34}$ highlight the need to invest further in the provision of urgent dental care beyond COVID-19 and to increase the number of UDC-type services in order to accommodate patients with acute dental conditions who struggle to access a dentist. ${ }^{35}$

Our participants' experiences also highlighted existing problems within healthcare systems, such as the lack of transferability of medical records and information between dentistry and other healthcare sectors (that is, GPs and hospitals) and the impact that this can have on patient care. Sharing medical records and expanding prescribing infrastructures to include dentistry, by investing in efficient and secure IT systems, are necessary to improve care provision across sectors. Finally, despite the infrequent use of remote video consultations by our participants, patient satisfaction with such technologies was rated high in a study by Rahman et al..$^{36}$ Teledentistry could also be a viable option to effectively utilise staff who are shielding, are self-isolating or those with childcare responsibilities enabling them to continue to provide a service to patients remotely. However, more research is needed into the clinical effectiveness of remote working and how to overcome digital exclusion in certain population groups. ${ }^{36}$

\section{Strengths and weaknesses}

Given the diversity in UDC settings across England, our approach allowed diverse perspectives to be drawn, enhancing richness of data and transferability of findings. The analytical framework used to synthesise the findings enabled us to provide a rigorous analysis of data. The involvement of multiple researchers with diverse experiences and backgrounds in the analysis of data ensured credibility. By supporting the narrative descriptions with quotes and relevant context, confirmability was attained. To ensure dependability, the research and analytical process was clearly documented. All of the above ensured trustworthiness in our findings.
Non-clinical members of the dental team working in the UDC (for example, administrative and managerial staff) were not considered for participation. Triangulating our findings by interviewing all team members of the dental team, along with commissioners and decision-makers as well patients treated in UDCs, could have provided greater insight into the experiences of different stakeholders and enabled us to identify further potential areas of improvement.

\section{Conclusions}

This study has highlighted the dedication and endeavour of dental teams during the COVID19 pandemic, and the multiple challenges it presented. Participants experienced both emotional and physical challenges, exacerbated by an unsupported environment, often due to what was perceived as a lack of leadership. However, positive emotions and coping strategies were also identified. Consistent and supportive leadership is required to build trust with the dental profession in recovery. Collective and sustained efforts at system level to improve the mental wellbeing of the current and future dental workforce are timely. There is a need to develop robust national guidance for managing dental service in scenarios where routine dental care is restricted, alongside greater integration of dentistry into wider healthcare to optimise patient care and experience.

\section{Ethics declaration}

The authors declare no conflicts of interest.

\section{Acknowledgements}

The authors would like to thank our participants who engaged with our research and shared their views and experiences with us. We would also like to thank all organisations, the journals and individuals who kindly cascaded the information about our research study. Anastasios Plessas and Martha Paisi share first co-authorship as they contributed equally to the production of this paper. Funding: Peninsula Dental Social Enterprise (PDSE) partially funded this study.

\section{Author contributions}

Study conceptualisation: the study was conceptualised by MP, AP, RW and MBD. Interview guide: the interview guide was initially developed by $M P, A P$ and RW. The interview guide was then amended and finalised after receiving critical feedback by $M B D, H W$ and IM. Data collection: the interviews were conducted and transcribed verbatim by AP.
Data analysis: coding and identification of emerging themes - five interviews were independently coded by $A P, M B D$ and RB. Coding results were compared in a team meeting, overseen by an experienced qualitative researcher $(H W)$, and emerging themes were identified and discussed. $R B$, who has extensive qualitative research experience, then coded half of the interviews. Sense checking was performed to review the analysis and to question the rationale for decisions ( $R B, A P, H W, M P)$. Subsequently, all interviews were coded by $R B$ and codes and subthemes were discussed with HW, MP, AP and RW. Refining and defining of themes: finally, the emergent themes were generated, defined and named by AP, MP, HW and $R B$. Draft manuscript: the draft manuscript was jointly prepared by AP and MP. The manuscript was finalised after receiving critical feedback by $H W, R B$, $R W, M B D$ and IM.

\section{References}

1. World Health Organisation. WHO announces COVID-19 outbreak a pandemic. 2020. Available at https://www. euro.who.int/en/health-topics/health-emergencies/ coronavirus-covid-19/news/news/2020/3/whoannounces-covid-19-outbreak-a-pandemic (accessed July 2021).

2. Iacobucci G. Covid-19: UK lockdown is "crucial" to saving lives, say doctors and scientists. BMJ 2020; DOI: 10.1136/bmj.m1204.

3. Scottish Dental Clinical Effectiveness Programme. Rapid Review of Aerosol Generating Procedures in Dentistry. 2021. Available online at https://www.sdcep.org.uk/ published-guidance/covid-19-practice-recovery/rapidreview-of-agps/ (accessed July 2021).

4. Banakar M, Bagheri Lankarani K, Jafarpour D, Moayedi S, Banakar M H, MohammadSadeghi A. COVID-19 transmission risk and protective protocols in dentistry: a systematic review. BMC Oral Health 2020; 20: 275.

5. NHS England. Resumption of dental services in England. 2020. Available at https://www.england.nhs.uk/ coronavirus/wp-content/uploads/sites/52/2020/03/ Urgent-dental-care-letter-28-May.pdf (accessed July 2021)

6. NHS England. COVID-19 guidance and standard operating procedure: For the provision of urgent dental care in primary care dental settings and designated urgent dental care provider sites. 2020. Available at https:// www.england.nhs.uk/coronavirus/wp-content/uploads/ sites/52/2020/06/C0581-covid-19-urgent-dental-caresop-update-16-june-20-.pdf (accessed July 2021).

7. British Dental Association. Dentists: PPE shortages leaving staff at risk and urgent care system in jeopardy. 2020. Available at https://bda.org/news-centre/pressreleases/Pages/PPE-shortages-leaving-staff-at-riskand-urgent-care-system-in-jeopardy-.aspx (accessed July 2021).

8. Iheduru-Anderson K. Reflections on the lived experience of working with limited personal protective equipment during the COVID-19 crisis. Nurs Inq 2021; DOI: 10.1111/nin.12382

9. Vindrola-Padros C, Andrews L, Dowrick A et al. Perceptions and experiences of healthcare workers during the COVID-19 pandemic in the UK. BMJ Open 2020; DOI: 10.1136/bmjopen-2020-040503.

10. Eftekhar Ardebili M, Naserbakht M, Bernstein C, Alazmani-Noodeh F, Hakimi H, Ranjbar H. Healthcare providers experience of working during the COVID-19 pandemic: A qualitative study. Am J Infect Control 2021; 49: 547-554.

11. Aughterson $H$, McKinlay $A R$, Fancourt $D$, Burton $A$. Psychosocial impact on frontline health and social care professionals in the UK during the COVID-19 pandemic: a qualitative interview study. BMJ Open 2021; DOI: 10.1136/bmjopen-2020-047353. 
12. Holmes E A, O'Connor R C, Perry V H et al. Multidisciplinary research priorities for the COVID-19 pandemic: a call for action for mental health science. Lancet Psychiatry 2020; 7: 547-560.

13. Blackhall K K, Singh R P. Dental emergencies presenting to maxillofacial units during the COVID-19 pandemic: a five-centre UK hospital study. Br Dent J 2021; DOI: 10.1038/s41415-020-2499-1.

14. Carter E, Currie C C, Asuni A et al. The first six weeks setting up a UK urgent dental care centre during the COVID-19 pandemic. Br Dent J 2020; 228: 842-848.

15. Shah A, Bryant C, Patel J, Tagar H, Akintola D, Obisesan O. COVID-19: establishing an oral surgery-led urgent dental care hub. Br Dent J 2020; 228: 957-963.

16. Hammarberg K, Kirkman M, de Lacey S. Qualitative research methods: when to use them and how to judge them. Hum Reprod 2016; 31: 498-501.

17. Lin C S. Revealing the "Essence" of Things: Using Phenomenology in LIS Research. Qual Quant Methods Libraries 2017: 2: 469-478.

18. Braun V, Clarke V. Successful qualitative research: A practical guide for beginners. London: SAGE, 2013.

19. Marshall M N. Sampling for qualitative research. Fam Pract 1996; 13: 522-526.

20. Ritchie J, Lewis J, Elam G. Designing and selecting samples. In Ritchie J, Lewis J (eds) Qualitative Research Practice. pp 77-108. London: SAGE, 2003.

21. Barbour R. Introducing qualitative research: a student's guide. 2nd ed. London: SAGE, 2014.

22. Braun $V$, Clarke V. Using thematic analysis in psychology. Qual Res Psychol 2006; 3: 101-177.
23. Swain J. A Hybrid Approach to Thematic Analysis in Qualitative Research: Using a Practical Example. London: SAGE, 2018.

24. Nyashanu M, Pfende F, Ekpenyong M. Exploring the challenges faced by frontline workers in health and social care amid the COVID-19 pandemic: experiences of frontline workers in the English Midlands region, UK. J Interprof Care 2020; 34: 655-661.

25. Vera San Juan N, Aceituno D, Djellouli N et al. Mental health and well-being of healthcare workers during the COVID-19 pandemic in the UK: contrasting guidelines with experiences in practice. BJPsych Open 2020; DOI: 10.1192/bjo.2020.148

26. Collin V, Toon M, O'Selmo E, Reynolds L, Whitehead $P$. A survey of stress, burnout and well-being in UK dentists. Br Dent J 2019; 226: 40-49.

27. Mahendran K, Patel S, Sproat C. Psychosocial effects of the COVID-19 pandemic on staff in a dental teaching hospital. Br Dent J 2020; 229: 127-132.

28. Liu Q, Luo D, Haase J E et al. The experiences of health-care providers during the COVID-19 crisis in China: a qualitative study. Lancet Glob Health 2020; DOI: 10.1016/S2214-109X(20)30204-7.

29. Collin V, O'Selmo E, Whitehead P. Psychological distress and the perceived impact of the COVID-19 pandemic on UK dentists during a national lockdown. Br Dent J 2021; DOI: 10.1038/s41415-020-2592-5.

30. Fujiwara D, Dolan P, Lawton R et al. The Wellbeing Costs of COVID-19 in the UK. 2020. Available at https://www.jacobs.com/sites/default/ files/2020-05/jacobs-wellbeing-costs-of-covid19-uk.pdf (accessed July 2021).

31. Dental Professional Alliance. Mental Health Wellness in Dentistry. 2021. Available online at https://mhwd. org/download/mental-health-wellness-in-dentistryframework/ (accessed July 2021).

32. Plessas A, Paisi M, Bryce M et al. Mental Health and Wellbeing in Dentistry: A Rapid Evidence Assessment. 2021. Available online at https://www. gdc-uk.org/about-us/what-we-do/research/ourresearch-library/detail/report/mental-health-andwellbeing-in-dentistry-a-rapid-evidence-assessment (accessed July 2021).

33. Shah S, Wordley V, Thompson W. How did COVID-19 impact on dental antibiotic prescribing across England? Br Dent J 2020; 229: 601-604.

34. Healthwatch England. What people are telling us: A summary July-September 2020. 2020. Available at https://www.healthwatch.co.uk/sites/healthwatch. co.uk/files/20201208\%20A\%20review\%20of\%20 our\%20evidence\%20Q2\%202020-21.pdf (accessed July 2021).

35. NHS England and NHS Improvement. Commissioning Standard for Urgent Dental Care. 2019. Available at https://www.england.nhs.uk/wp-content/ uploads/2019/07/commissioning-standard-forurgent-dental-care.pdf (accessed July 2021).

36. Rahman N, Nathwani S, Kandiah T. Teledentistry from a patient perspective during the coronavirus pandemic. Br Dent J 2020; DOI: 10.1038/ s41415-020-1919-6. 